\title{
First Results from Simultaneous Dual Frequency Observations of Pulsars
}

\author{
Y. Gupta, P. Gothoskar and N.D. Ramesh Bhat \\ National Centre for Radio Astrophysics, TIFR, Post Bag 3, \\ Ganeshkhind, Pune 411007, India
}

\begin{abstract}
One of the unique capabilities of the GMRT is the facility for simultaneous multi-frequency observations of pulsars. We have initiated such observations with the aims of (i) studying frequency dependence of pulsar emission, (ii) accurate estimates of pulsar dispersion measure and its variations and (iii) multi-frequency interstellar scintillation studies. Here we present some results from the ongoing dual frequency observations of pulsars.
\end{abstract}

\section{Introduction}

The Giant Metrewave Radio Telescope (GMRT), nearing completion at Khodad near Pune in India, is going to be a major facility for research in radio astronomy (Swarup et al. 1997). The GMRT consists of 30 antennas, each of 45 metre diameter, spread out over a radius of $15 \mathrm{~km}$. The GMRT is designed to operate in six frequency bands: $50 \mathrm{MHz}, 150 \mathrm{MHz}, 235 \mathrm{MHz}, 325 \mathrm{MHz}, 610 \mathrm{MHz}$ and 1000 - $1450 \mathrm{MHz}$.

The GMRT has two main modes of operation: (i) aperture synthesis mode for mapping radio sources and (ii) array mode for study of compact objects such as pulsars. In the array mode, the signals from the different antennas can be combined in incoherent or coherent mode, depending on the requirements. In the incoherent mode, the pulsar receiver provides total power output from 256 spectral channels spanning a maximum bandwidth of $16 \mathrm{MHz}$ for each of two orthogonal polarizations, at a minimum sampling time of 16 microseconds.

Besides providing a large effective collecting area $(\approx 30,000$ sq.m. $)$ for pulsar observations, one of the special capabilities of the GMRT is the ability to observe simultaneously at two (or more) different wave-bands. This is achieved by grouping the antennas into sub-arrays, each operating at a different frequency, and suitably processing the signals from these.

\section{Dual Frequency Observations}

Simultaneous multi frequency observations of pulsars are useful from many points of view. At the GMRT, we have started a program for dual frequency monitoring of several pulsars, with the following goals in mind: 
(i) Pulsar emission studies: Both single pulses and average profiles show significant changes with frequency of observation. Here, simultaneous observations of pulsar signals at a range of radio frequencies can play an important role in improving our understanding of the mechanism by which the pulses are generated.

(ii) Accurate estimation of dispersion measure (DM): Our data can be used to obtain very accurate estimates of the dispersion measure. For a sample of bright pulsars, we have been able to achieve an accuracy of better than 1 part in $10^{4}$. We can thus study DM fluctuations with time which gives a direct estimate the spectrum of electron density fluctuations in the ISM.

(iii) Interstellar scintillation (ISS) studies: Since ISS is a frequency dependent phenomena, simultaneous dual frequency observations of pulsar dynamic spectra can be used as a powerful tool to study ISS.

In this paper, we concentrate on one example of pulsar emission studies using simultaneous dual frequency observations of pulsars with the GMRT.

\section{Data Analysis and Results}

The simultaneous dual frequency observations described here were carried out using 12 antennas in the incoherent array mode of the GMRT. Of these 5 were configured for the $238 \mathrm{MHz}$ band and 7 for the $610 \mathrm{MHz}$ band, with bandwidth of $8 \mathrm{MHz}$ (with 128 spectral channels) and $16 \mathrm{MHz}$ (with 256 spectral channels), respectively. The powers from the two circular polarizations at each observing band were added to get the total signal. The pulsar dispersion was used to discriminate between and separate out the data for these two frequencies during off-line analysis. This is possible whenever the combination of pulsar dispersion measure, period and pulse shape produces non-overlapping dispersion tracks in the frequency time plane. Thus, the data were dedispersed for the two frequency bands and the resulting time serieses were aligned by removing the dispersion delay between the center frequencies of the two frequency bands.

Results from observations of the pulsar PSR B0329+54 are treated in detail here. The data analysed had 1500 simultaneous pulses at the two frequencies. The average profiles show the expected shapes, with the core component and two conal components clearly visible. In particular, the increase of the overall pulse width at the lower frequency due to the increase in shift of the conal components with respect to the core, is clearly seen (figure 1, top curve). This is commonly understood to be due to radius-to-frequency mapping (i.e. lower frequencies being emitted at larger heights but on the same magnetic field lines) in the pulsar magnetosphere (Cordes, 1978). To investigate this effect, we have looked at the longitude of occurrence of the conal components for the individual pulses. Here, we find that the longitude shift between the conal components at the two frequencies varies over a significant range from pulse to pulse. There are several pulses which show shifts that are quite different from the shifts in the average profile (see figure 1 for some examples). Such a behaviour is not expected from the canonical radius-to-frequency mapping model. 
To investigate this quantitatively, we have computed the cross-correlation function (CCF) between the corresponding conal components at the two frequencies, for the average profile, as well as for each individual pulse. Some of these are shown in the right panel of figure 1, where the peak of the CCF for the average profile is indicated by the two solid lines (one for each conal component) and the arrows indicate the peak of the CCF for individual pulses. In figure 2, we show the histograms of the locations of the CCF peaks for the two conal components. The data is for components that exceed a given noise threshold for the peak value. The dashed lines indicate the location of the peak of the $\mathrm{CCF}$ for the average profiles. The peak of the histograms are significantly offset from the dashed lines, confirming that many pulses show longitudinal shifts of the conal components that are significantly different from that seen for the average profiles. There is also some evidence that the discrepancy in the shift is proportional to the strength of the conal components in the individual pulses, though this is not conclusively established.

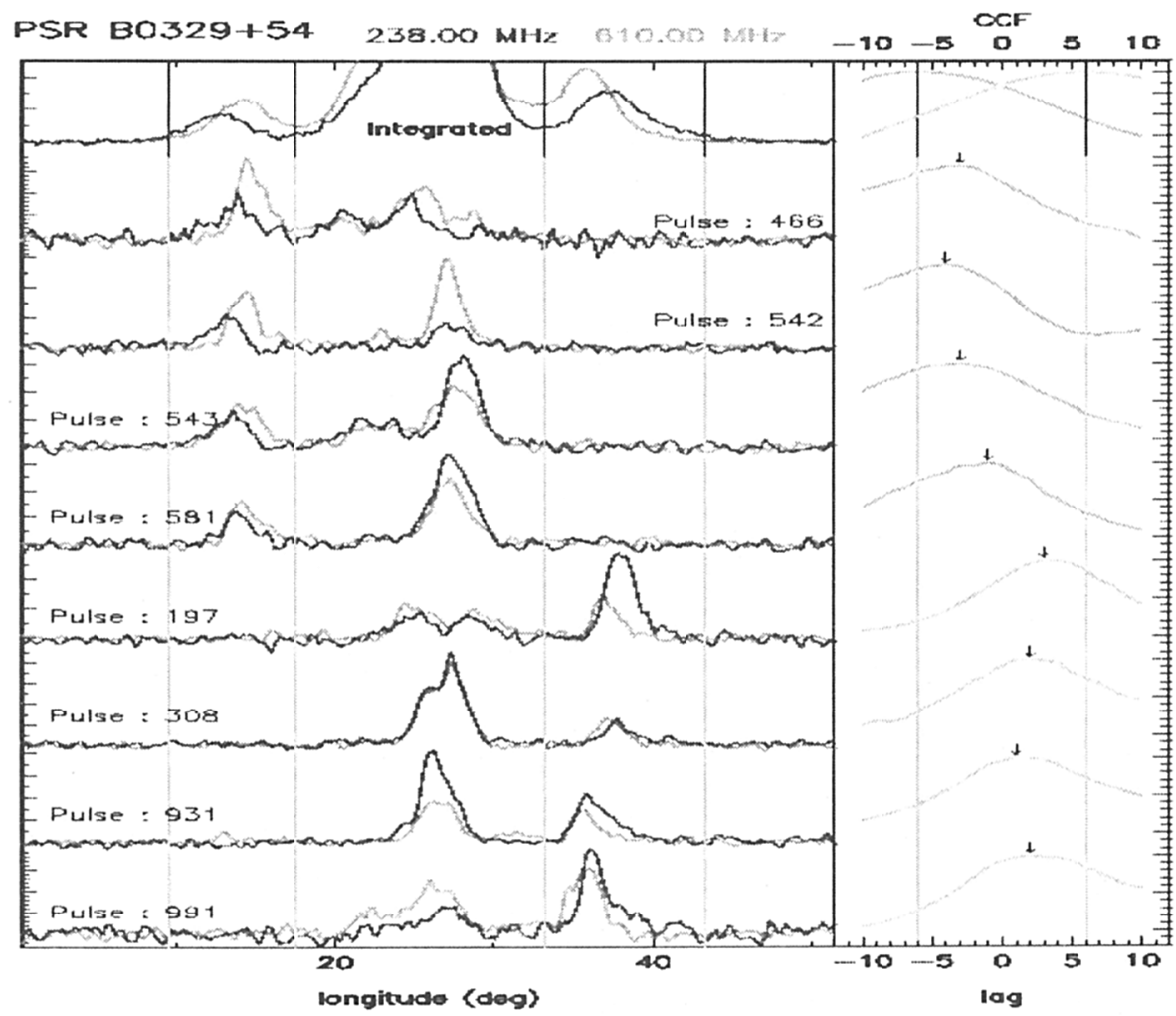

Figure 1. Average profile (top curve) and selected single pulses at 238 and $610 \mathrm{MHz}$ for PSR $\mathrm{B} 0329+54$. The average profile plot is clipped at the top to show the conal components in detail. The panel on the right shows the CCFs for the conal components. 

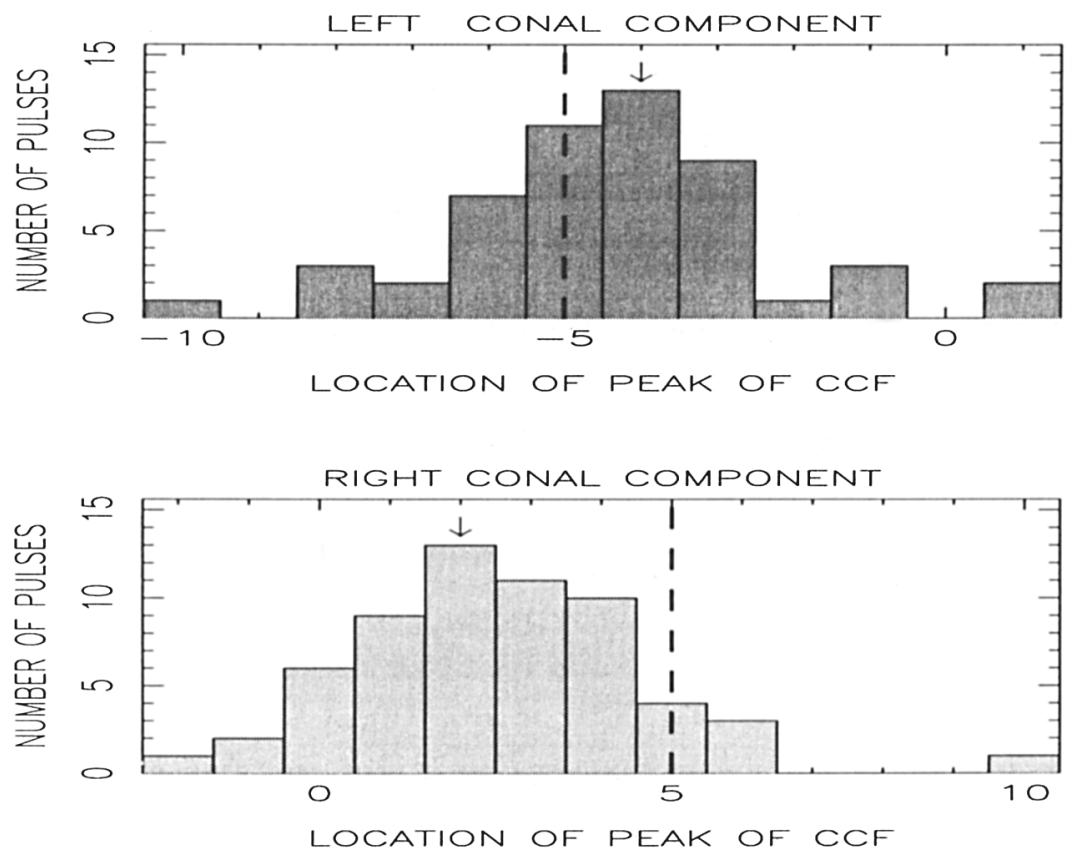

Figure 2. Histograms for the distribution of the peaks of the CCFs for the left and right conal components for individual pulses.

\section{Conclusions}

We have shown that GMRT is an ideal instrument for simultaneous multifrequency observations of radio pulsars. Such observations are a powerful means of studying pulse emission properties, dispersion measure variations and interstellar scintillations. Our analysis of simultaneous single pulse observations of PSR B0329+54 at 238 and $610 \mathrm{MHz}$ shows that the observed separation of conal components for strong single pulses at these two frequencies does not appear to be consistent with the radius-to-frequency mapping effect seen in the average profiles. Further investigations of this should produce interesting insights to the pulsar emission mechanism.

\section{References}

Swarup, G., Ananthakrishnan, S., Subrahmanya, C.R., Rao, A.P., Kulkarni, V.K., \& Kapahi, V.K. 1997, in High Sensitivity Radio Astronomy, ed. N. Jackson \& R..J. Davis (Cambridge University Press)

Cordes. J.M. 1978, Ap.J, 222, 1006 